\title{
Roundness error evaluation of cold embossed hole based on profile measurement technique
}

\begin{abstract}
Roundness is one of the main features to ensure the perfect mating of assembled parts. A coordinate measurement machine is commonly utilized for measurement, but the use of this machine is limited because of the small size and characteristics of the hole. This study aims to evaluate the quality of an AA6061 cold embossing hole. A simple approach based on profile deviation is proposed to evaluate the roundness of the hole. The profile is obtained with the use of InfiniteFocus Alicona system, a commercially available 3D surface measurement technique. Several specimens with various depths and diameters are prepared for measurement. Results are validated with the commercial Mitutoyo roundtest machine.
\end{abstract}

Keyword: Roundness; Cold embossing; Profile measurement 